\title{
On solving variational inequality problems involving quasi- monotone operators via modified Tseng's extragradient methods with convergence analysis
}

\author{
Nopparat Wairojjana ${ }^{\mathrm{a}}$, Nuttapol Pakkaranang ${ }^{\mathrm{b}}$, Supansa Noinakorn ${ }^{\mathrm{b}, *}$ \\ ${ }^{a}$ Applied Mathematics Program, Faculty of Science and Technology, Valaya Alongkorn Rajabhat University under the Royal Patronage, \\ 1 Moo 20 Phaholyothin Road, Klong Neung, Klong Luang, Pathumthani 13180, Thailand. \\ ${ }^{b}$ Mathematics and Computing Science Program, Faculty of Science and Technology, Phetchabun Rajabhat University, Phetchabun \\ 67000, Thailand.
}

\begin{abstract}
The main objective of this research is to find the numerical solution of variational inequalities involving quasimonotone operators in infinite-dimensional real Hilbert spaces. The main advantage of these iterative schemes is that they allow the uncomplicated calculation of step size rules that depend on the knowledge of an operator explanation instead of the Lipschitz constant or some other line search method. The proposed iterative schemes follow a monotone and non-monotone step size procedure based on mapping (operator) information as a replacement for its Lipschitz constant or some other line search method. The strong convergences are well proven, analogous to the proposed methods, and impose certain control specification conditions. Finally, to verify the effectiveness of the iterative methods, we present some numerical experiments.
\end{abstract}

Keywords: Variational inequality problem, Tseng's extragradient method, strong convergence theorems, quasimonotone operator, Lipschitz continuity.

2020 MSC: 47J25, 47H09, 47H06, 47J05.

(C2022 All rights reserved.

\section{Introduction}

The main objective of this paper is to investigate the iterative methods used to estimate the solution to the variational inequality problem [26] in a real Hilbert space involving quasimonotone operators. Assume that $\Sigma$ is a real Hilbert space and $\Delta$ is a nonempty, closed, and convex subset of $\Sigma$. Consider the operator $\Upsilon: \Sigma \rightarrow \Sigma$. The variational inequality problem for $\Upsilon$ on $\Delta$ is defined in the following manner:

$$
\text { Find } \omega^{*} \in \Delta \text { such that }\left\langle\curlyvee\left(\omega^{*}\right), y-\omega^{*}\right\rangle \geqslant 0, \forall y \in \Delta \text {. }
$$

The mathematical model of the variational inequality problem is a key problem in nonlinear analysis. It is a significant mathematical model that unifies a number of crucial concepts in applied mathematics, such as a nonlinear system of equations, optimization conditions for problems with the optimization

\footnotetext{
${ }^{*}$ Corresponding author

Email addresses: nopparat@vru.ac.th (Nopparat Wairojjana), nuttapol.pak@pcru.ac.th (Nuttapol Pakkaranang), supansa.noi@pcru.ac.th (Supansa Noinakorn)
}

doi: $10.22436 /$ jmcs.027.01.04

Received: 2021-11-29 Revised: 2021-12-17 Accepted: 2022-01-06 
process, complementarity problems, network equilibrium problems, and finance (see for more details $[13,15-18])$. As a consequence, this notion has various applications in the fields of mathematical programming, engineering, transport analysis, network economics, game theory, and computer science. The regularized method and the projection method are two prominent and general procedures for finding a solution to variational inequalities. It is also noted that the first approach is most commonly used to deal with the variational inequalities accompanied by the class of monotone operators. The regularized sub-problem in this method is strongly monotone, and its unique solution is found more conveniently than the initial problem. In this study, we discuss the projection methods that are well known for their simpler numerical computing. In addition, projection methods are useful for approximating the numerical solution of variational inequalities. Many researchers have developed various projection methods to solve such problems (see for more details $[4,6,7,10,11,14,19,22,24,28,35,36,39]$ ) and others in $[3,5,8,9,12,20,23,25,29-33,37,38,40]$. Almost all methods for solving the problem (VIP) are based on the computation of a projection on the feasible set $\Delta$. Korpelevich [19] and Antipin [1] introduced the following extragradient method. Their method takes the following form:

$$
\left\{\begin{array}{l}
u_{1} \in \Delta, \\
y_{n}=P_{\Delta}\left[u_{n}-\varkappa \Upsilon\left(u_{n}\right)\right], \\
u_{n+1}=P_{\Delta}\left[u_{n}-\varkappa \Upsilon\left(y_{n}\right)\right]
\end{array}\right.
$$

where $0<\varkappa<\frac{1}{\mathrm{~L}}$. Given the above method, we have used two projections on the underlying set $\Delta$ for each iteration. This, of course, can affect the computational effectiveness of the method if the feasible set $\Delta$ has a complicated structure. Here, we present some methods that can overcome this drawback. The first is the following subgradient extragradient method introduced by Censor et al. [10]. This method takes the following form:

$$
\left\{\begin{array}{l}
u_{1} \in \Delta, \\
y_{n}=P_{\Delta}\left[u_{n}-\varkappa \Upsilon\left(u_{n}\right)\right] \\
u_{n+1}=P_{\Sigma_{n}}\left[u_{n}-\varkappa \curlyvee\left(y_{n}\right)\right]
\end{array}\right.
$$

where $0<\varkappa<\frac{1}{\mathrm{~L}}$ and

$$
\Sigma_{n}=\left\{z \in \Sigma:\left\langle u_{n}-\varkappa \Upsilon\left(u_{n}\right)-y_{n}, z-y_{n}\right\rangle \leqslant 0\right\}
$$

In this article, our main focus on the Tseng's extragradient method [28] that uses only one projection for each iteration. This method takes the following form:

$$
\left\{\begin{array}{l}
u_{1} \in \Delta, \\
y_{n}=P_{\Delta}\left[u_{n}-\varkappa \Upsilon\left(u_{n}\right)\right], \\
u_{n+1}=y_{n}+\varkappa\left[\Upsilon\left(u_{n}\right)-\Upsilon\left(y_{n}\right)\right],
\end{array}\right.
$$

where $0<\varkappa<\frac{1}{\mathrm{~L}}$. It is important to note that the above-mentioned methods have two major flaws: a fixed constant step size rule that is dependent on the Lipschitz constant of mapping and generates a weakly convergent iterative sequence. The Lipschitz constant is generally unknown or difficult to compute. From a computational point of view, it can be difficult to consider a fixed step size constraint that affects the method's efficiency and rate of convergence. In addition, the study of a strongly convergent iterative sequence is important in the context of an infinite-dimensional Hilbert space.

A natural question has been raised:

"Is it possible to introduce new strongly convergent Tseng's extragradient-type method by using a monotonic and non-monotonic variable step size rule to solve variational inequalities involving quasimonotone operator?

This research aims to explore variational inequalities involving quasimonotone operators in infinitedimensional Hilbert spaces. Furthermore, to show that the iterative sequences generated by all four subgradient extragradient algorithms strongly converge to a solution. Both the monotone and non-monotone variable step size rules are used in subgradient and extragradient algorithms. The investigation of inertial 
algorithms is also presented, which usually improves the efficiency of the iterative sequence. The paper's key contribution is to investigate explicit monotone and non-monotone step size rules with inertial schemes and achieve strong convergence.

The paper is arranged in the following way. In Sect. 2, preliminary results were presented. Sect. 3 gives all new algorithms and their convergence analysis. Finally, Sect. 4 gives some numerical results to explain the practical efficiency of the proposed methods.

\section{Preliminaries}

This section contains a number of important identities and relevant lemmas. For any $u, y \in \Sigma$, we have

$$
\|u+y\|^{2}=\|u\|^{2}+2\langle u, y\rangle+\|y\|^{2} .
$$

A metric projection $\mathrm{P}_{\Delta}\left(\mathrm{y}_{1}\right)$ of $\mathrm{y}_{1} \in \Sigma$ is defined by

$$
P_{\Delta}\left(y_{1}\right)=\arg \min \left\{\left\|y_{1}-y_{2}\right\|: y_{2} \in \Delta\right\} .
$$

First, we list some of the important identities of projection mapping and others.

Lemma 2.1 ([2]). Let $\mathrm{P}_{\Delta}: \Sigma \rightarrow \Delta$ be a metric projection. For any $\mathrm{y}_{1}, \mathrm{y}_{2}, \mathrm{y}_{3} \in \Sigma$ and $\ell \in \mathbb{R}$, then, the following inequalities are hold:

(i) $\mathrm{y}_{3}=\mathrm{P}_{\Delta}\left(\mathrm{y}_{1}\right)$ if and only if

$$
\left\langle y_{1}-y_{3}, y_{2}-y_{3}\right\rangle \leqslant 0, \forall y_{2} \in \Delta ;
$$

(ii)

$$
\left\|y_{1}-P_{\Delta}\left(y_{2}\right)\right\|^{2}+\left\|P_{\Delta}\left(y_{2}\right)-y_{2}\right\|^{2} \leqslant\left\|y_{1}-y_{2}\right\|^{2}, y_{1} \in \Delta, y_{2} \in \Sigma ;
$$

$$
\left\|y_{1}-P_{\Delta}\left(y_{1}\right)\right\| \leqslant\left\|y_{1}-y_{2}\right\|, y_{2} \in \Delta, y_{1} \in \Sigma ;
$$

$$
\left\|\ell y_{1}+(1-\ell) y_{2}\right\|^{2}=\ell\left\|y_{1}\right\|^{2}+(1-\ell)\left\|y_{2}\right\|^{2}-\ell(1-\ell)\left\|y_{1}-y_{2}\right\|^{2}
$$

(v)

$$
\left\|y_{1}+y_{2}\right\|^{2} \leqslant\left\|y_{1}\right\|^{2}+2\left\langle y_{2}, y_{1}+y_{2}\right\rangle .
$$

Lemma 2.2 ([34]). Let $\left\{\boldsymbol{e}_{\mathrm{n}}\right\} \subset[0,+\infty)$ be a sequence satisfies the following condition

$$
e_{\mathrm{n}+1} \leqslant\left(1-f_{n}\right) e_{n}+f_{n} g_{n}, \forall n \in \mathbb{N} .
$$

In addition, two sequences $\left\{\mathrm{f}_{\mathrm{n}}\right\} \subset(0,1)$ and $\left\{\mathrm{g}_{\mathrm{n}}\right\} \subset \mathbb{R}$ satisfy the following conditions:

$$
\lim _{n \rightarrow+\infty} f_{n}=0, \sum_{n=1}^{+\infty} f_{n}=+\infty \text { and } \limsup _{n \rightarrow+\infty} g_{n} \leqslant 0 .
$$

Then, $\lim _{n \rightarrow+\infty} e_{n}=0$.

Lemma 2.3 ([21]). Let $\left\{e_{n}\right\} \subset \mathbb{R}$ be a sequence and there exists a subsequence $\left\{n_{i}\right\}$ of $\{n\}$ such that

$$
e_{n_{i}}<e_{n_{i+1}}, \forall i \in \mathbb{N} .
$$

Then, there exists a nondecreasing sequence $\mathrm{m}_{\mathrm{k}} \subset \mathbb{N}$ such that $\mathrm{m}_{\mathrm{k}} \rightarrow+\infty$ as $\mathrm{k} \rightarrow+\infty$, with

$$
e_{m_{k}} \leqslant e_{m_{k+1}} \text { and } e_{k} \leqslant e_{m_{k+1}}, \forall k \in \mathbb{N} .
$$

Indeed, $m_{k}=\max \left\{j \leqslant k: e_{j} \leqslant e_{j+1}\right\}$. 


\section{Main results}

In this section, we propose a class of iterative algorithms based on Tseng's extragradient method for solving quasimonotone variational inequalities. The methods are all described in detail in the following text. The following conditions are assumed to be met in order to confirm the strong convergence.

$(\curlyvee 1)$ The solution set for problem (VIP) is denoted by $\mathrm{VI}(\Delta, \Upsilon)$ is nonempty.

$(\Upsilon 2)$ An operator $\Upsilon: \Sigma \rightarrow \Sigma$ is said to be quasimonotone if

$$
\langle\curlyvee(u), y-u\rangle>0 \Longrightarrow\langle\curlyvee(y), y-u\rangle \geqslant 0, \forall u, y \in \Delta \text {. }
$$

$(\curlyvee 3)$ An operator $\Upsilon: \Sigma \rightarrow \Sigma$ is said to be Lipschitz continuous if there exists a constant $L>0$ such that

$$
\|\curlyvee(\mathrm{u})-\Upsilon(\mathrm{y})\| \leqslant \mathrm{L}\|\mathrm{u}-\mathrm{y}\|, \forall \mathrm{u}, \mathrm{y} \in \Delta .
$$

$(\curlyvee 4)$ An operator $\Upsilon: \Sigma \rightarrow \Sigma$ is sequentially weakly continuous if $\left\{\curlyvee\left(u_{n}\right)\right\}$ weakly converges to $\Upsilon(u)$ for every sequence $\left\{u_{n}\right\}$ weakly converges to $u$.

Now, we are in a position to propose a new variant of the extragradient method to solve quasimonotone variational inequalities in real Hilbert spaces and prove a strong convergence result for the proposed method.

Algorithm 1 (Halpern extragradient method with fixed step size rule)

STEP 0: Let $u_{1} \in \Delta, 0<\varkappa<\frac{1}{\mathrm{~L}}$ and $\left\{\vartheta_{n}\right\} \subset(0,1)$ meet the following conditions:

$$
\lim _{n \rightarrow+\infty} \vartheta_{n}=0 \text { and } \sum_{n=1}^{+\infty} \vartheta_{n}=+\infty \text {. }
$$

STEP 1: Compute

$$
y_{n}=P_{\Delta}\left(u_{n}-\varkappa \Upsilon\left(u_{n}\right)\right) .
$$

If $u_{n}=y_{n}$, STOP. Otherwise, go to STEP 2.

STEP 2: Compute

$$
z_{n}=y_{n}+\varkappa\left[\Upsilon\left(u_{n}\right)-\Upsilon\left(y_{n}\right)\right]
$$

STEP 3: Compute

$$
u_{n+1}=\vartheta_{n} u_{1}+\left(1-\vartheta_{n}\right) z_{n} .
$$

Set $n:=n+1$ and go back to STEP 1 .

Lemma 3.1. Suppose that $\Upsilon: \Sigma \rightarrow \Sigma$ satisfies the conditions $(\curlyvee 1)-(\Upsilon 4)$ and sequence $\left\{u_{n}\right\}$ generated by Algorithm 1. Then, we have

$$
\left\|u_{n+1}-\omega^{*}\right\|^{2} \leqslant\left\|u_{n}-\omega^{*}\right\|^{2}-\left(1-\varkappa^{2} L^{2}\right)\left\|u_{n}-y_{n}\right\|^{2} .
$$

Proof. Since $\omega^{*} \in \operatorname{VI}(\Delta, \Upsilon)$, we have

$$
\begin{aligned}
& \left\|u_{n+1}-\omega^{*}\right\|^{2}=\left\|y_{n}+\varkappa\left[\Upsilon\left(u_{n}\right)-\Upsilon\left(y_{n}\right)\right]-\omega^{*}\right\|^{2} \\
& =\left\|y_{n}-\omega^{*}\right\|^{2}+\varkappa^{2}\left\|\Upsilon\left(u_{n}\right)-\Upsilon\left(y_{n}\right)\right\|^{2}+2 \varkappa\left\langle y_{n}-\omega^{*}, \Upsilon\left(u_{n}\right)-\Upsilon\left(y_{n}\right)\right\rangle \\
& =\left\|y_{n}+u_{n}-u_{n}-\omega^{*}\right\|^{2}+\varkappa^{2}\left\|\Upsilon\left(u_{n}\right)-\Upsilon\left(y_{n}\right)\right\|^{2}+2 \varkappa\left\langle y_{n}-\omega^{*}, \curlyvee\left(u_{n}\right)-\curlyvee\left(y_{n}\right)\right\rangle \\
& =\left\|y_{n}-u_{n}\right\|^{2}+\left\|u_{n}-\omega^{*}\right\|^{2}+2\left\langle y_{n}-u_{n}, u_{n}-\omega^{*}\right\rangle \\
& +\varkappa^{2}\left\|\Upsilon\left(u_{n}\right)-\Upsilon\left(y_{n}\right)\right\|^{2}+2 \varkappa\left\langle y_{n}-\omega^{*}, \Upsilon\left(u_{n}\right)-\Upsilon\left(y_{n}\right)\right\rangle \\
& =\left\|u_{n}-\omega^{*}\right\|^{2}+\left\|y_{n}-u_{n}\right\|^{2}+2\left\langle y_{n}-u_{n}, y_{n}-\omega^{*}\right\rangle+2\left\langle y_{n}-u_{n}, u_{n}-y_{n}\right\rangle \\
& +\varkappa^{2}\left\|\Upsilon\left(u_{n}\right)-\Upsilon\left(y_{n}\right)\right\|^{2}+2 \varkappa\left\langle y_{n}-\omega^{*}, \Upsilon\left(u_{n}\right)-\Upsilon\left(y_{n}\right)\right\rangle \text {. }
\end{aligned}
$$

It is given that $y_{n}=P_{\Delta}\left[u_{n}-\varkappa \curlyvee\left(u_{n}\right)\right]$ and it gives that 


$$
\left\langle u_{n}-\varkappa \curlyvee\left(u_{n}\right)-y_{n}, y-y_{n}\right\rangle \leqslant 0, \forall y \in \Delta
$$

Thus, we have

$$
\left\langle u_{n}-y_{n}, \omega^{*}-y_{n}\right\rangle \leqslant \varkappa\left\langle\curlyvee\left(u_{n}\right), w^{*}-y_{n}\right\rangle .
$$

Combining expressions (3.1) and (3.2), we have

$$
\begin{aligned}
\left\|u_{n+1}-\omega^{*}\right\|^{2} \leqslant & \left\|u_{n}-\omega^{*}\right\|^{2}+\left\|y_{n}-u_{n}\right\|^{2}+2 \varkappa\left\langle\curlyvee\left(u_{n}\right), w^{*}-y_{n}\right\rangle-2\left\langle u_{n}-y_{n}, u_{n}-y_{n}\right\rangle \\
& +\varkappa^{2}\left\|\Upsilon\left(u_{n}\right)-\Upsilon\left(y_{n}\right)\right\|^{2}-2 \varkappa\left\langle\curlyvee\left(u_{n}\right)-\Upsilon\left(y_{n}\right), \omega^{*}-y_{n}\right\rangle \\
= & \left\|u_{n}-\omega^{*}\right\|^{2}-\left\|u_{n}-y_{n}\right\|^{2}+\varkappa^{2}\left\|\Upsilon\left(u_{n}\right)-\Upsilon\left(y_{n}\right)\right\|^{2}-2 \varkappa\left\langle\curlyvee\left(y_{n}\right), y_{n}-\omega^{*}\right\rangle .
\end{aligned}
$$

It is given that $\omega^{*}$ is the solution of the problem (VIP) implies that

$$
\left\langle\curlyvee\left(\omega^{*}\right), y-\omega^{*}\right\rangle \geqslant 0, \forall y \in \Delta \text {. }
$$

It implies that

$$
\left\langle\curlyvee(y), y-\omega^{*}\right\rangle \geqslant 0, \forall y \in \Delta
$$

By substituting $y=y_{n} \in \Delta$, we have

$$
\left\langle\curlyvee\left(y_{n}\right), y_{n}-\omega^{*}\right\rangle \geqslant 0
$$

From expressions (3.3) and (3.4), we obtain

$$
\begin{aligned}
\left\|u_{n+1}-w^{*}\right\|^{2} & \leqslant\left\|u_{n}-w^{*}\right\|^{2}-\left\|u_{n}-y_{n}\right\|^{2}+\varkappa^{2} L^{2}\left\|u_{n}-y_{n}\right\|^{2} \\
& =\left\|u_{n}-w^{*}\right\|^{2}-\left(1-\varkappa^{2} L^{2}\right)\left\|u_{n}-y_{n}\right\|^{2} .
\end{aligned}
$$

Next, we introduce a variant of Algorithm 1 in which the constant step size $\varkappa$ is chosen adaptively and thus produced a sequence $\varkappa_{n}$ that does not require the knowledge of the Lipschitz-type constants L.

Algorithm 2 (Monotonic explicit Halpern extragradient method with variable step size rule)

STEP 0: Let $u_{1} \in \Delta, \varkappa_{1}>0, x \in(0,1)$ and $\left\{\vartheta_{n}\right\} \subset(0,1)$ meet the following conditions:

$$
\lim _{n \rightarrow+\infty} \vartheta_{n}=0 \text { and } \sum_{n=1}^{+\infty} \vartheta_{n}=+\infty
$$

STEP 1: Compute

$$
y_{n}=P_{\Delta}\left(u_{n}-\varkappa_{n} \Upsilon\left(u_{n}\right)\right) \text {. }
$$

If $u_{n}=y_{n}$, STOP. Otherwise, go to STEP 2.

STEP 2: Compute

$$
z_{n}=y_{n}+\varkappa_{n}\left[\Upsilon\left(u_{n}\right)-\Upsilon\left(y_{n}\right)\right]
$$

STEP 3: Compute $u_{n+1}=\vartheta_{n} u_{1}+\left(1-\vartheta_{n}\right) z_{n}$.

STEP 4: Compute

$$
\varkappa_{n+1}= \begin{cases}\min \left\{\varkappa_{n}, \frac{x\left\|u_{n}-y_{n}\right\|}{\left\|\Upsilon\left(u_{n}\right)-\Upsilon\left(y_{n}\right)\right\|}\right\}, & \text { if } \Upsilon\left(u_{n}\right)-\Upsilon\left(y_{n}\right) \neq 0, \\ \varkappa_{n}, & \text { otherwise. }\end{cases}
$$

Set $n:=n+1$ and go back to STEP 1 .

Lemma 3.2. The sequence $\left\{\varkappa_{n}\right\}$ generated by (3.5) is decreasing monotonically and converges to $\varkappa>0$. 
Proof. It is given that $\Upsilon$ is Lipschitz-continuous with constant $L>0$. Let $\Upsilon\left(u_{n}\right) \neq \Upsilon\left(y_{n}\right)$ such that

$$
\frac{x\left\|u_{n}-y_{n}\right\|}{\left\|\Upsilon\left(u_{n}\right)-\Upsilon\left(y_{n}\right)\right\|} \geqslant \frac{x\left\|u_{n}-y_{n}\right\|}{L\left\|u_{n}-y_{n}\right\|} \geqslant \frac{x}{L}
$$

The above expression implies that $\lim _{n \rightarrow+\infty} \varkappa_{n}=\varkappa$.

Now, we propose a second variant of the first method to solve quasimonotone variational inequalities in real Hilbert spaces and prove a strong convergence result for the proposed method. The second method involves a non-monotonic self-adaptive step rule to make the method independent of the Lipschitz constant. The second method is written as follows.

Algorithm 3 (Non-monotonic explicit Halpern extragradient method with variable step size rule)

STEP 0: Let $u_{1} \in \Delta, \varkappa_{1}>0, \chi \in(0,1)$ and sequence $\left\{\varphi_{n}\right\}$ satisfying $\sum_{n=1}^{+\infty} \varphi_{n}<+\infty$. Moreover, $\left\{\vartheta_{n}\right\} \subset(0,1)$ satisfying the following conditions:

$$
\lim _{n \rightarrow+\infty} \vartheta_{n}=0 \text { and } \sum_{n=1}^{+\infty} \vartheta_{n}=+\infty
$$

STEP 1: Compute

$$
y_{n}=P_{\Delta}\left(u_{n}-\varkappa_{n} \Upsilon\left(u_{n}\right)\right) .
$$

If $u_{n}=y_{n}$, STOP. Otherwise, go to STEP 2.

STEP 2: Compute $z_{n}=y_{n}+\varkappa_{n}\left[\Upsilon\left(u_{n}\right)-\Upsilon\left(y_{n}\right)\right]$.

STEP 3: Compute $u_{n+1}=\vartheta_{n} u_{1}+\left(1-\vartheta_{n}\right) z_{n}$.

STEP 4: Compute

$$
\varkappa_{n+1}= \begin{cases}\min \left\{\varkappa_{n}+\varphi_{n}, \frac{x\left\|u_{n}-y_{n}\right\|}{\left\|\Upsilon\left(u_{n}\right)-\Upsilon\left(y_{n}\right)\right\|}\right\}, & \text { if } \Upsilon\left(u_{n}\right)-\Upsilon\left(y_{n}\right) \neq 0, \\ \varkappa_{n}+\varphi_{n}, & \text { otherwise. }\end{cases}
$$

Set $n:=n+1$ and go back to STEP 1 .

Lemma 3.3. A sequence $\left\{\varkappa_{n}\right\}$ generated by (3.6) is convergent to $\varkappa$ and satisfying the following inequality

$$
\min \left\{\frac{\chi}{\mathrm{L}}, \varkappa_{1}\right\} \leqslant \varkappa_{n} \leqslant \varkappa_{1}+\mathrm{P} \text { where } \mathrm{P}=\sum_{n=1}^{+\infty} \varphi_{n} .
$$

Proof. It is given that $\Upsilon$ is Lipschitz-continuous with constant $L>0$. Let $\Upsilon\left(u_{n}\right) \neq \Upsilon\left(y_{n}\right)$ such that

$$
\frac{x\left\|u_{n}-y_{n}\right\|}{\left\|\Upsilon\left(u_{n}\right)-\Upsilon\left(y_{n}\right)\right\|} \geqslant \frac{x\left\|u_{n}-y_{n}\right\|}{L\left\|u_{n}-y_{n}\right\|} \geqslant \frac{x}{L}
$$

By using mathematical induction on the definition of $\varkappa_{n+1}$, we have

$$
\min \left\{\frac{X}{L}, \varkappa_{1}\right\} \leqslant \varkappa_{n} \leqslant \varkappa_{1}+P .
$$

Let $\left[\varkappa_{n+1}-\varkappa_{n}\right]^{+}=\max \left\{0, \varkappa_{n+1}-\varkappa_{n}\right\}$ and $\left[\varkappa_{n+1}-\varkappa_{n}\right]^{-}=\max \left\{0,-\left(\varkappa_{n+1}-\varkappa_{n}\right)\right\}$. From the definition of $\left\{\varkappa_{n}\right\}$, we have

$$
\sum_{n=1}^{+\infty}\left(\varkappa_{n+1}-\varkappa_{n}\right)^{+}=\sum_{n=1}^{+\infty} \max \left\{0, \varkappa_{n+1}-\varkappa_{n}\right\} \leqslant P<+\infty .
$$


That is, the series $\sum_{n=1}^{+\infty}\left(\varkappa_{n+1}-\varkappa_{n}\right)^{+}$is convergent. Next, we need to prove the convergence of $\sum_{n=1}^{+\infty}\left(\varkappa_{n+1}-\right.$ $\left.\varkappa_{n}\right)^{-}$. Let $\sum_{n=1}^{+\infty}\left(\varkappa_{n+1}-\varkappa_{n}\right)^{-}=+\infty$. Due to the reason that $\varkappa_{n+1}-\varkappa_{n}=\left(\varkappa_{n+1}-\varkappa_{n}\right)^{+}-\left(\varkappa_{n+1}-\varkappa_{n}\right)^{-}$, thus, we have

$$
\varkappa_{k+1}-\varkappa_{1}=\sum_{n=0}^{k}\left(\varkappa_{n+1}-\varkappa_{n}\right)=\sum_{n=0}^{k}\left(\varkappa_{n+1}-\varkappa_{n}\right)^{+}-\sum_{n=0}^{k}\left(\varkappa_{n+1}-\varkappa_{n}\right)^{-} .
$$

By allowing $k \rightarrow+\infty$ in (3.7), we have $\varkappa_{k} \rightarrow-\infty$ as $k \rightarrow+\infty$. This is a contradiction. Due to the convergence of the series $\sum_{n=0}^{k}\left(\varkappa_{n+1}-\varkappa_{n}\right)^{+}$and $\sum_{n=0}^{k}\left(\varkappa_{n+1}-\varkappa_{n}\right)^{-}$taking $k \rightarrow+\infty$ in (3.7), we obtain $\lim _{n \rightarrow+\infty} \varkappa_{n}=\varkappa$. This completes the proof.

Lemma 3.4. Assume that $\Upsilon: \Sigma \rightarrow \Sigma$ satisfies the conditions $(\curlyvee 1)-(\Upsilon 4)$. Let $\left\{u_{n}\right\}$ be a sequence generated by Algorithms 2 and 3. For each $\omega^{*} \in \mathrm{VI}(\Delta, \curlyvee)$, we have

$$
\left\|u_{n+1}-\omega^{*}\right\|^{2} \leqslant\left\|u_{n}-\omega^{*}\right\|^{2}-\left(1-\chi^{2} \frac{\varkappa_{n}^{2}}{x_{n+1}^{2}}\right)\left\|u_{n}-y_{n}\right\|^{2} .
$$

Proof. Let $\omega^{*} \in \operatorname{VI}(\Delta, \Upsilon)$ and by definition of $u_{n+1}$, we have

$$
\begin{aligned}
\left\|u_{n+1}-\omega^{*}\right\|^{2}= & \left\|y_{n}+\varkappa_{n}\left[\Upsilon\left(u_{n}\right)-\Upsilon\left(y_{n}\right)\right]-\omega^{*}\right\|^{2} \\
= & \left\|y_{n}-\omega^{*}\right\|^{2}+\varkappa_{n}^{2}\left\|\Upsilon\left(u_{n}\right)-\Upsilon\left(y_{n}\right)\right\|^{2}+2 \varkappa_{n}\left\langle y_{n}-w^{*}, \Upsilon\left(u_{n}\right)-\Upsilon\left(y_{n}\right)\right\rangle \\
= & \left\|y_{n}+u_{n}-u_{n}-\omega^{*}\right\|^{2}+\varkappa_{n}^{2}\left\|\Upsilon\left(u_{n}\right)-\Upsilon\left(y_{n}\right)\right\|^{2}+2 \varkappa_{n}\left\langle y_{n}-\omega^{*}, \Upsilon\left(u_{n}\right)-\Upsilon\left(y_{n}\right)\right\rangle \\
= & \left\|y_{n}-u_{n}\right\|^{2}+\left\|u_{n}-\omega^{*}\right\|^{2}+2\left\langle y_{n}-u_{n}, u_{n}-\omega^{*}\right\rangle \\
& +\varkappa_{n}^{2}\left\|\Upsilon\left(u_{n}\right)-\Upsilon\left(y_{n}\right)\right\|^{2}+2 \varkappa_{n}\left\langle y_{n}-\omega^{*}, \Upsilon\left(u_{n}\right)-\Upsilon\left(y_{n}\right)\right\rangle \\
= & \left\|u_{n}-\omega^{*}\right\|^{2}+\left\|y_{n}-u_{n}\right\|^{2}+2\left\langle y_{n}-u_{n}, y_{n}-\omega^{*}\right\rangle+2\left\langle y_{n}-u_{n}, u_{n}-y_{n}\right\rangle \\
& +\varkappa_{n}^{2}\left\|\Upsilon\left(u_{n}\right)-\Upsilon\left(y_{n}\right)\right\|^{2}+2 \varkappa_{n}\left\langle y_{n}-\omega^{*}, \Upsilon\left(u_{n}\right)-\Upsilon\left(y_{n}\right)\right\rangle .
\end{aligned}
$$

It is given that $y_{n}=P_{\Delta}\left[u_{n}-\varkappa_{n} \Upsilon\left(u_{n}\right)\right]$ and it further implies that

$$
\left\langle u_{n}-\varkappa_{n} \Upsilon\left(u_{n}\right)-y_{n}, y-y_{n}\right\rangle \leqslant 0, \forall y \in \Delta .
$$

Moreover, equivalently for some $\omega^{*} \in \mathrm{VI}(\Delta, \Upsilon)$, we can write

$$
\left\langle u_{n}-y_{n}, \omega^{*}-y_{n}\right\rangle \leqslant \varkappa_{n}\left\langle\curlyvee\left(u_{n}\right), \omega^{*}-y_{n}\right\rangle .
$$

Combining expressions (3.8) and (3.9), we have

$$
\begin{aligned}
\left\|u_{n+1}-\omega^{*}\right\|^{2} \leqslant & \left\|u_{n}-w^{*}\right\|^{2}+\left\|y_{n}-u_{n}\right\|^{2}+2 \varkappa_{n}\left\langle\curlyvee\left(u_{n}\right), w^{*}-y_{n}\right\rangle-2\left\langle u_{n}-y_{n}, u_{n}-y_{n}\right\rangle \\
& +\varkappa_{n}^{2}\left\|\Upsilon\left(u_{n}\right)-\Upsilon\left(y_{n}\right)\right\|^{2}-2 \varkappa_{n}\left\langle\curlyvee\left(u_{n}\right)-\Upsilon\left(y_{n}\right), \omega^{*}-y_{n}\right\rangle \\
= & \left\|u_{n}-\omega^{*}\right\|^{2}-\left\|u_{n}-y_{n}\right\|^{2}+\varkappa_{n}^{2}\left\|\Upsilon\left(u_{n}\right)-\Upsilon\left(y_{n}\right)\right\|^{2}-2 \varkappa_{n}\left\langle\curlyvee\left(y_{n}\right), y_{n}-\omega^{*}\right\rangle .
\end{aligned}
$$

It is given that $\omega^{*}$ is the solution of the problem (VIP) implying that

$$
\left\langle\curlyvee\left(\omega^{*}\right), y-\omega^{*}\right\rangle>0, \forall y \in \Delta \text {. }
$$


Due to the property of $\Upsilon$ on $\Delta$, we obtain

$$
\left\langle\curlyvee(y), y-w^{*}\right\rangle \geqslant 0, \forall y \in \Delta
$$

Substituting $y=y_{n} \in \Delta$, we have

$$
\left\langle\curlyvee\left(y_{n}\right), y_{n}-\omega^{*}\right\rangle \geqslant 0
$$

Combining expressions (3.10) and (3.11) we obtain

$$
\begin{aligned}
\left\|u_{n+1}-\omega^{*}\right\|^{2} & \leqslant\left\|u_{n}-w^{*}\right\|^{2}-\left\|u_{n}-y_{n}\right\|^{2}+x^{2} \frac{x_{n}^{2}}{x_{n+1}^{2}}\left\|u_{n}-y_{n}\right\|^{2} \\
& =\left\|u_{n}-\omega^{*}\right\|^{2}-\left(1-\chi^{2} \frac{x_{n}^{2}}{x_{n+1}^{2}}\right)\left\|u_{n}-y_{n}\right\|^{2} .
\end{aligned}
$$

Lemma 3.5. Let $\Upsilon: \Sigma \rightarrow \Sigma$ be an operator satisfying the conditions $(\Upsilon 1)-(\Upsilon 4)$. If there exists a subsequence $\left\{u_{n_{k}}\right\}$ weakly convergent to $\hat{u}$ and $\lim _{k \rightarrow+\infty}\left\|u_{n_{k}}-y_{n_{k}}\right\|=0$, then, $\hat{u} \in \operatorname{VI}(\Delta, \curlyvee)$.

Proof. Since $\left\{u_{n_{k}}\right\}$ is weakly convergent to $\hat{u}$ and due to $\lim _{k \rightarrow+\infty}\left\|u_{n_{k}}-y_{n_{k}}\right\|=0$, the sequence $\left\{y_{n_{k}}\right\}$ also is weakly convergent to $\hat{u}$. Next, we need to prove that $\hat{u} \in \operatorname{VI}(\Delta, \curlyvee)$. By value of $y_{n}$, we have

$$
y_{n_{k}}=P_{\Delta}\left[u_{n_{k}}-\varkappa_{n_{k}} \Upsilon\left(u_{n_{k}}\right)\right]
$$

that is equivalent to

$$
\left\langle u_{n_{k}}-\varkappa_{n_{k}} \Upsilon\left(u_{n_{k}}\right)-y_{n_{k}}, y-y_{n_{k}}\right\rangle \leqslant 0, \forall y \in \Delta .
$$

The above inequality implies that

$$
\left\langle u_{n_{k}}-y_{n_{k}}, y-y_{n_{k}}\right\rangle \leqslant \varkappa_{n_{k}}\left\langle\curlyvee\left(u_{n_{k}}\right), y-y_{n_{k}}\right\rangle, \forall y \in \Delta .
$$

Thus, we obtain

$$
\frac{1}{\varkappa_{n_{k}}}\left\langle u_{n_{k}}-y_{n_{k}}, y-y_{n_{k}}\right\rangle+\left\langle\curlyvee\left(u_{n_{k}}\right), y_{n_{k}}-u_{n_{k}}\right\rangle \leqslant\left\langle\curlyvee\left(u_{n_{k}}\right), y-u_{n_{k}}\right\rangle, \forall y \in \Delta .
$$

By the use of $\lim _{k \rightarrow+\infty}\left\|u_{n_{k}}-y_{n_{k}}\right\|=0$ and $k \rightarrow+\infty$ in expression (3.12), we have

$$
\liminf _{k \rightarrow+\infty}\left\langle\curlyvee\left(u_{n_{k}}\right), y-u_{n_{k}}\right\rangle \geqslant 0, \forall y \in \Delta .
$$

Furthermore, it implies that

$$
\left\langle\curlyvee\left(y_{n_{k}}\right), y-y_{n_{k}}\right\rangle=\left\langle\curlyvee\left(y_{n_{k}}\right)-\Upsilon\left(u_{n_{k}}\right), y-u_{n_{k}}\right\rangle+\left\langle\curlyvee\left(u_{n_{k}}\right), y-u_{n_{k}}\right\rangle+\left\langle\curlyvee\left(y_{n_{k}}\right), u_{n_{k}}-y_{n_{k}}\right\rangle .
$$

Since $\lim _{k \rightarrow+\infty}\left\|u_{n_{k}}-y_{n_{k}}\right\|=0$, thus, we have

$$
\lim _{k \rightarrow+\infty}\left\|\Upsilon\left(u_{n_{k}}\right)-\Upsilon\left(y_{n_{k}}\right)\right\|=0,
$$

which together with expressions (3.13) and (3.14), we obtain

$$
\liminf _{k \rightarrow+\infty}\left\langle\curlyvee\left(y_{n_{k}}\right), y-y_{n_{k}}\right\rangle \geqslant 0, \forall y \in \Delta .
$$

Moreover, let us take a positive sequence $\left\{\epsilon_{k}\right\}$ that is decreasing and convergent to zero. For each $\left\{\epsilon_{k}\right\}$ there exists a least positive integer denoted by $m_{k}$ such that

$$
\left\langle\curlyvee\left(u_{n_{i}}\right), y-u_{n_{i}}\right\rangle+\epsilon_{k}>0, \forall i \geqslant m_{k} .
$$


Since $\left\{\epsilon_{k}\right\}$ is decreasing sequence and it is easy to see that the sequence $\left\{m_{k}\right\}$ is increasing. If there exists a natural number $N_{0} \in \mathbb{N}$ such that for all $\Upsilon\left(u_{n_{m_{k}}}\right) \neq 0, n_{m_{k}} \geqslant N_{0}$. Consider that

$$
\Gamma_{n_{m_{k}}}=\frac{\Upsilon\left(u_{n_{m_{k}}}\right)}{\left\|\Upsilon\left(u_{n_{m_{k}}}\right)\right\|^{2}}, \forall n_{m_{k}} \geqslant N_{0} .
$$

Due to the above definition, we have

$$
\left\langle\curlyvee\left(u_{n_{m_{k}}}\right), \Gamma_{n_{m_{k}}}\right\rangle=1, \forall n_{m_{k}} \geqslant N_{0} .
$$

Moreover, from expressions (3.15) and (3.16) for all $n_{m_{k}} \geqslant N_{0}$, we have

$$
\left\langle\curlyvee\left(u_{n_{m_{k}}}\right), y+\epsilon_{k} \Gamma_{n_{m_{k}}}-u_{n_{m_{k}}}\right\rangle>0
$$

By the definition of quasimonotone, we have

$$
\left\langle\curlyvee\left(y+\epsilon_{k} \Gamma_{n_{m_{k}}}\right), y+\epsilon_{k} \Gamma_{n_{m_{k}}}-u_{n_{m_{k}}}\right\rangle>0
$$

For all $n_{m_{k}} \geqslant N_{0}$, we have

$$
\left\langle\curlyvee(y), y-u_{n_{m_{k}}}\right\rangle \geqslant\left\langle\curlyvee(y)-\Upsilon\left(y+\epsilon_{k} \Gamma_{n_{m_{k}}}\right), y+\epsilon_{k} \Gamma_{n_{m_{k}}}-u_{n_{m_{k}}}\right\rangle-\epsilon_{k}\left\langle\curlyvee(y), \Gamma_{n_{m_{k}}}\right\rangle .
$$

Due to $\left\{u_{n_{k}}\right\}$ converges weakly to $\hat{u} \in \Delta$ with $\Upsilon$ is weakly sequentially continuous on the set $\Delta$ we obtain $\left\{\curlyvee\left(u_{n_{k}}\right)\right\}$ converges weakly to $\Upsilon(\hat{u})$. Let $\Upsilon(\hat{u}) \neq 0$ implies that

$$
\|\Upsilon(\hat{u})\| \leqslant \liminf _{k \rightarrow+\infty}\left\|\Upsilon\left(u_{n_{k}}\right)\right\|
$$

Since $\left\{u_{n_{m_{k}}}\right\} \subset\left\{u_{n_{k}}\right\}$ and $\lim _{k \rightarrow+\infty} \epsilon_{k}=0$, we have

$$
0 \leqslant \lim _{k \rightarrow+\infty}\left\|\epsilon_{k} \Gamma_{n_{m_{k}}}\right\|=\lim _{k \rightarrow+\infty} \frac{\epsilon_{k}}{\left\|\Upsilon\left(u_{n_{m_{k}}}\right)\right\|} \leqslant \frac{0}{\|\Upsilon(\hat{u})\|}=0 .
$$

By letting $k \rightarrow+\infty$ in (3.17), we obtain

$$
\langle\curlyvee(y), y-\hat{u}\rangle \geqslant 0, \forall y \in \Delta .
$$

Let $u \in \Delta$ be an arbitrary element and for $0<\varkappa \leqslant 1$, let

$$
\hat{u}_{\varkappa}=\varkappa u+(1-\varkappa) \hat{u} \text {. }
$$

Then $\hat{u}_{\varkappa} \in \Delta$ and from (3.18) we have

$$
\varkappa\left\langle\curlyvee\left(\hat{u}_{\varkappa}\right), u-\hat{u}\right\rangle \geqslant 0 .
$$

Hence

$$
\left\langle\curlyvee\left(\hat{\mathrm{u}}_{\varkappa}\right), \mathrm{u}-\hat{\mathrm{u}}\right\rangle \geqslant 0 .
$$

Let $\varkappa \rightarrow 0$. Then $\hat{u}_{\varkappa} \rightarrow \hat{u}$ along a line segment. By the continuity of an operator, $\Upsilon\left(\hat{u}_{\varkappa}\right)$ converges to $\Upsilon(\hat{u})$ as $\varkappa \rightarrow 0$. It follows from (3.19) that

$$
\langle\curlyvee(\hat{u}), u-\hat{u}\rangle \geqslant 0 .
$$

Therefore $\hat{u}$ is a solution of problem (VIP).

Theorem 3.6. Assume that a sequence $\left\{u_{n}\right\}$ generated by Algorithm 3 and $\omega^{*} \in \mathrm{VI}(\Delta, \Upsilon)$. Then, $\left\{u_{n}\right\}$ converges strongly to $\omega^{*}=\mathrm{P}_{\mathrm{VI}(\Delta, \Upsilon)}\left(\mathrm{u}_{1}\right)$. 
Proof. Since $\varkappa_{n} \rightarrow \varkappa$ there exists a positive number $\epsilon \in\left(0,1-\chi^{2}\right)$ such that

$$
\lim _{n \rightarrow+\infty}\left(1-\frac{\chi^{2} \varkappa_{n}^{2}}{\varkappa_{n+1}^{2}}\right)=1-\chi^{2}>\epsilon>0
$$

Thus, there exists a finite number $n_{1} \in \mathbb{N}$ such that

$$
\left(1-\frac{\chi^{2} \varkappa_{n}^{2}}{\varkappa_{n+1}^{2}}\right)>\epsilon>0, \forall n \geqslant n_{1}
$$

Hence, by Lemma 3.4, we obtain

$$
\left\|z_{n}-\omega^{*}\right\|^{2} \leqslant\left\|u_{n}-\omega^{*}\right\|^{2}, \forall n \geqslant n_{1} .
$$

Since $\omega^{*} \in \operatorname{VI}(\Delta, \Upsilon)$ and by the use of definition of $\left\{u_{n+1}\right\}$, we have

$$
\begin{aligned}
\left\|u_{n+1}-\omega^{*}\right\| & =\left\|\vartheta_{n} u_{1}+\left(1-\vartheta_{n}\right) z_{n}-\omega^{*}\right\| \\
& =\left\|\vartheta_{n}\left[u_{1}-\omega^{*}\right]+\left(1-\vartheta_{n}\right)\left[z_{n}-\omega^{*}\right]\right\| \leqslant \vartheta_{n}\left\|u_{1}-\omega^{*}\right\|+\left(1-\vartheta_{n}\right)\left\|z_{n}-\omega^{*}\right\| .
\end{aligned}
$$

Combining expressions (3.20) with (3.21) and $\vartheta_{n} \subset(0,1)$, we have

$$
\begin{aligned}
\left\|u_{n+1}-\omega^{*}\right\| & \leqslant \vartheta_{n}\left\|u_{1}-\omega^{*}\right\|+\left(1-\vartheta_{n}\right)\left\|u_{n}-\omega^{*}\right\| . \\
& \leqslant \max \left\{\left\|u_{1}-\omega^{*}\right\|,\left\|u_{n}-\omega^{*}\right\|\right\} \leqslant \max \left\{\left\|u_{1}-\omega^{*}\right\|,\left\|u_{n_{1}}-\omega^{*}\right\|\right\} .
\end{aligned}
$$

Thus, we conclude that the $\left\{u_{n}\right\}$ is a bounded sequence. Next, we need to prove the strong convergence of the iterative sequence $\left\{u_{n}\right\}$ generated by Algorithm 3. The Lipschitz continuity property indicates that the solution set $\mathrm{VI}(\Delta, \curlyvee)$ is a convex and closed set (see for details [27]). Let $\omega^{*}=P_{V I(\Delta, \Upsilon)}\left(u_{1}\right)$ and by Lemma 2.1 (i) we have

$$
\left\langle u_{1}-\omega^{*}, y-\omega^{*}\right\rangle \leqslant 0, \forall y \in \operatorname{VI}(\Delta, \Upsilon)
$$

By using Lemma 2.1 (iv), we obtain

$$
\begin{aligned}
\left\|u_{n+1}-\omega^{*}\right\|^{2}= & \left\|\vartheta_{n} u_{1}+\left(1-\vartheta_{n}\right) z_{n}-\omega^{*}\right\|^{2} \\
= & \left\|\vartheta_{n}\left[u_{1}-\omega^{*}\right]+\left(1-\vartheta_{n}\right)\left[z_{n}-\omega^{*}\right]\right\|^{2} \\
= & \vartheta_{n}\left\|u_{1}-\omega^{*}\right\|^{2}+\left(1-\vartheta_{n}\right)\left\|z_{n}-\omega^{*}\right\|^{2}-\vartheta_{n}\left(1-\vartheta_{n}\right)\left\|u_{1}-z_{n}\right\|^{2} \\
\leqslant & \vartheta_{n}\left\|u_{1}-\omega^{*}\right\|^{2}+\left(1-\vartheta_{n}\right)\left[\left\|u_{n}-\omega^{*}\right\|^{2}-\left(1-\frac{\chi^{2} \varkappa_{n}^{2}}{\varkappa_{n+1}^{2}}\right)\left\|u_{n}-y_{n}\right\|^{2}\right] \\
& -\vartheta_{n}\left(1-\vartheta_{n}\right)\left\|u_{1}-z_{n}\right\|^{2} \\
\leqslant & \vartheta_{n}\left\|u_{1}-\omega^{*}\right\|^{2}+\left\|u_{n}-\omega^{*}\right\|^{2}-\left(1-\vartheta_{n}\right)\left(1-\frac{\chi^{2} \varkappa_{n}^{2}}{\varkappa_{n+1}^{2}}\right)\left\|u_{n}-y_{n}\right\|^{2} .
\end{aligned}
$$

The above expression implies that

$$
\left(1-\vartheta_{n}\right)\left(1-\frac{\chi^{2} \varkappa_{n}^{2}}{\varkappa_{n+1}^{2}}\right)\left\|u_{n}-y_{n}\right\|^{2} \leqslant \vartheta_{n}\left\|u_{1}-\omega^{*}\right\|^{2}+\left\|u_{n}-\omega^{*}\right\|^{2}-\left\|u_{n+1}-\omega^{*}\right\|^{2} .
$$

The remainder of the proof shall be splited into the following two parts.

Case 1: Assume that there exists a fixed number $n_{2} \in \mathbb{N}$ such that

$$
\left\|u_{n+1}-\omega^{*}\right\| \leqslant\left\|u_{n}-\omega^{*}\right\|, \forall n \geqslant n_{2} .
$$


Thus, above implies that $\lim _{n \rightarrow+\infty}\left\|u_{n}-\omega^{*}\right\|$ exists and let $\lim _{n \rightarrow+\infty}\left\|u_{n}-\omega^{*}\right\|=l$. From expression (3.22) we have

$$
\left(1-\vartheta_{n}\right)\left(1-\frac{\chi^{2} \varkappa_{n}^{2}}{\varkappa_{n+1}^{2}}\right)\left\|u_{n}-y_{n}\right\|^{2} \leqslant \vartheta_{n}\left\|u_{1}-\omega^{*}\right\|^{2}+\left\|u_{n}-\omega^{*}\right\|^{2}-\left\|u_{n+1}-\omega^{*}\right\|^{2} .
$$

By existence of $\lim _{n \rightarrow+\infty}\left\|u_{n}-\omega^{*}\right\|=l$ and $\vartheta_{n} \rightarrow 0$, we can deduce that

$$
\lim _{n \rightarrow+\infty}\left\|u_{n}-y_{n}\right\|=0 \text {. }
$$

Furthermore, we have

$$
\left\|z_{n}-y_{n}\right\|=\left\|y_{n}+\varkappa\left[\Upsilon\left(u_{n}\right)-\Upsilon\left(y_{n}\right)\right]-y_{n}\right\| \leqslant \varkappa L\left\|u_{n}-y_{n}\right\|
$$

It follows that

$$
\lim _{n \rightarrow+\infty}\left\|u_{n}-z_{n}\right\| \leqslant \lim _{n \rightarrow+\infty}\left\|u_{n}-y_{n}\right\|+\lim _{n \rightarrow+\infty}\left\|y_{n}-z_{n}\right\|=0
$$

Furthermore, we obtain

$$
\begin{aligned}
\left\|u_{n+1}-u_{n}\right\| & =\left\|\vartheta_{n} u_{1}+\left(1-\vartheta_{n}\right) z_{n}-u_{n}\right\| \\
& =\left\|\vartheta_{n}\left[u_{1}-u_{n}\right]+\left(1-\vartheta_{n}\right)\left[z_{n}-u_{n}\right]\right\| \leqslant \vartheta_{n}\left\|u_{1}-u_{n}\right\|+\left(1-\vartheta_{n}\right)\left\|z_{n}-u_{n}\right\| .
\end{aligned}
$$

It follows that

$$
\lim _{n \rightarrow+\infty}\left\|u_{n+1}-u_{n}\right\|=0 .
$$

Thus, the implies that the sequences $\left\{y_{n}\right\}$ and $\left\{z_{n}\right\}$ are bounded. Due to the reflexivity of $\Sigma$ and the boundedness of $\left\{u_{n}\right\}$ guarantees that there exists a subsequence $\left\{u_{n_{k}}\right\}$ such that $\left\{u_{n_{k}}\right\} \rightarrow \hat{u} \in \Sigma$ as $k \rightarrow$ $+\infty$. Next, we need to prove that $\hat{u} \in \operatorname{VI}(\Delta, \curlyvee)$. By Lemma 3.4, it follows that $\hat{u} \in \operatorname{VI}(\Delta, \Upsilon)$. Next, consider that

$$
\limsup _{n \rightarrow+\infty}\left\langle u_{1}-\omega^{*}, u_{n}-\omega^{*}\right\rangle=\limsup _{k \rightarrow+\infty}\left\langle u_{1}-\omega^{*}, u_{n_{k}}-\omega^{*}\right\rangle=\left\langle u_{1}-\omega^{*}, \hat{u}-\omega^{*}\right\rangle \leqslant 0
$$

By the use of $\lim _{n \rightarrow+\infty}\left\|u_{n+1}-u_{n}\right\|=0$, we may deduce that

$$
\limsup _{n \rightarrow+\infty}\left\langle u_{1}-\omega^{*}, u_{n+1}-\omega^{*}\right\rangle \leqslant \limsup _{n \rightarrow+\infty}\left\langle u_{1}-w^{*}, u_{n+1}-u_{n}\right\rangle+\limsup _{n \rightarrow+\infty}\left\langle u_{1}-w^{*}, u_{n}-w^{*}\right\rangle \leqslant 0 .
$$

By using Lemma $2.1(\mathrm{v})$, we have

$$
\begin{aligned}
\left\|u_{n+1}-\omega^{*}\right\|^{2} & =\left\|\vartheta_{n} u_{1}+\left(1-\vartheta_{n}\right) z_{n}-\omega^{*}\right\|^{2} \\
& =\left\|\vartheta_{n}\left[u_{1}-\omega^{*}\right]+\left(1-\vartheta_{n}\right)\left[z_{n}-\omega^{*}\right]\right\|^{2} \\
& \leqslant\left(1-\vartheta_{n}\right)^{2}\left\|z_{n}-\omega^{*}\right\|^{2}+2 \vartheta_{n}\left\langle u_{1}-\omega^{*},\left(1-\vartheta_{n}\right)\left[z_{n}-\omega^{*}\right]+\vartheta_{n}\left[u_{1}-\omega^{*}\right]\right\rangle \\
& =\left(1-\vartheta_{n}\right)^{2}\left\|z_{n}-\omega^{*}\right\|^{2}+2 \vartheta_{n}\left\langle u_{1}-\omega^{*}, u_{n+1}-\omega^{*}\right\rangle \\
& \leqslant\left(1-\vartheta_{n}\right)\left\|u_{n}-\omega^{*}\right\|^{2}+2 \vartheta_{n}\left\langle u_{1}-\omega^{*}, u_{n+1}-\omega^{*}\right\rangle .
\end{aligned}
$$

From expressions (3.25), (3.26), and using Lemma 2.2, we may deduce that $\left\|u_{n}-\omega^{*}\right\| \rightarrow 0$ as $n \rightarrow+\infty$.

Case 2: Assume that there exists a subsequence $\left\{n_{i}\right\}$ of $\{n\}$ such that

$$
\left\|u_{n_{i}}-\omega^{*}\right\| \leqslant\left\|u_{n_{i+1}}-\omega^{*}\right\|, \forall i \in \mathbb{N} .
$$

Thus, by Lemma 2.3, there exists a sequence $\left\{m_{k}\right\} \subset \mathbb{N}$ as $\left\{m_{k}\right\} \rightarrow+\infty$, such that

$$
\left\|u_{m_{k}}-\omega^{*}\right\| \leqslant\left\|u_{m_{k+1}}-\omega^{*}\right\| \text { and }\left\|u_{k}-\omega^{*}\right\| \leqslant\left\|u_{m_{k+1}}-\omega^{*}\right\|, \text { for all } k \in \mathbb{N} \text {. }
$$


As similar to the Case 1, expression (3.23) provides that

$$
\left(1-\vartheta_{m_{k}}\right)\left(1-\frac{\chi^{2} \varkappa_{m_{k}}^{2}}{\varkappa_{m_{k}+1}^{2}}\right)\left\|u_{m_{k}}-y_{m_{k}}\right\|^{2} \leqslant \vartheta_{m_{k}}\left\|u_{1}-\omega^{*}\right\|^{2}+\left\|u_{m_{k}}-\omega^{*}\right\|^{2}-\left\|u_{m_{k}+1}-\omega^{*}\right\|^{2} .
$$

Due to $\vartheta_{m_{k}} \rightarrow 0$, we deduce the following:

$$
\lim _{k \rightarrow+\infty}\left\|u_{m_{k}}-y_{m_{k}}\right\|=0 .
$$

It follows that

$$
\left\|z_{m_{k}}-y_{m_{k}}\right\|=\left\|y_{m_{k}}+\varkappa\left[\Upsilon\left(u_{m_{k}}\right)-\Upsilon\left(y_{m_{k}}\right)\right]-y_{m_{k}}\right\| \leqslant \varkappa L\left\|u_{m_{k}}-y_{m_{k}}\right\| .
$$

Furthermore, we have

$$
\lim _{k \rightarrow+\infty}\left\|u_{m_{k}}-z_{m_{k}}\right\| \leqslant \lim _{k \rightarrow+\infty}\left\|u_{m_{k}}-y_{m_{k}}\right\|+\lim _{k \rightarrow+\infty}\left\|y_{m_{k}}-z_{m_{k}}\right\|=0 .
$$

Also, we can obtain

$$
\begin{aligned}
\left\|u_{m_{k}+1}-u_{m_{k}}\right\| & =\left\|\vartheta_{m_{k}} u_{1}+\left(1-\vartheta_{m_{k}}\right) z_{m_{k}}-u_{m_{k}}\right\| \\
& =\left\|\vartheta_{m_{k}}\left[u_{1}-u_{m_{k}}\right]+\left(1-\vartheta_{m_{k}}\right)\left[z_{m_{k}}-u_{m_{k}}\right]\right\| \\
& \leqslant \vartheta_{m_{k}}\left\|u_{1}-u_{m_{k}}\right\|+\left(1-\vartheta_{m_{k}}\right)\left\|z_{m_{k}}-u_{m_{k}}\right\| \longrightarrow 0 .
\end{aligned}
$$

We use the same argument as in Case 1, which is as follows:

$$
\limsup _{k \rightarrow+\infty}\left\langle u_{1}-\omega^{*}, u_{m_{k}+1}-\omega^{*}\right\rangle \leqslant 0 .
$$

Now, using expressions (3.26) and (3.27), we have

$$
\begin{aligned}
\left\|u_{m_{k}+1}-\omega^{*}\right\|^{2} & \leqslant\left(1-\vartheta_{m_{k}}\right)\left\|u_{m_{k}}-\omega^{*}\right\|^{2}+2 \vartheta_{m_{k}}\left\langle u_{1}-\omega^{*}, u_{m_{k}+1}-\omega^{*}\right\rangle \\
& \leqslant\left(1-\vartheta_{m_{k}}\right)\left\|u_{m_{k}+1}-\omega^{*}\right\|^{2}+2 \vartheta_{m_{k}}\left\langle u_{1}-\omega^{*}, u_{m_{k}+1}-\omega^{*}\right\rangle .
\end{aligned}
$$

It continues from that

$$
\left\|u_{m_{k}+1}-\omega^{*}\right\|^{2} \leqslant 2\left\langle u_{1}-\omega^{*}, u_{m_{k}+1}-\omega^{*}\right\rangle .
$$

Since $\vartheta_{m_{k}} \rightarrow 0$ and $\left\|u_{m_{k}}-\omega^{*}\right\|$ is bounded, thus, by expressions (3.28) and (3.29) we obtain

$$
\left\|u_{m_{k}+1}-\omega^{*}\right\|^{2} \rightarrow 0, \text { as } k \rightarrow+\infty .
$$

It implies that

$$
\lim _{n \rightarrow+\infty}\left\|u_{k}-\omega^{*}\right\|^{2} \leqslant \lim _{n \rightarrow+\infty}\left\|u_{m_{k}+1}-\omega^{*}\right\|^{2} \leqslant 0 .
$$

Consequently, $u_{n} \rightarrow \omega^{*}$. This completes the proof of the theorem.

\section{Numerical illustration}

This section describes the numerical performance of the proposed algorithms, in contrast to some related work in the literature, as well as the analysis of how variations in control parameters affect the numerical effectiveness of the proposed algorithms. All computations are done in MATLAB R2018b and run on HP i-5 Core(TM)i5-6200 8.00 GB (7.78 GB usable) RAM laptop. 
Example 4.1. Let $\Sigma=l_{2}$ be a real Hilbert space with with the sequences of real numbers satisfying the following condition

$$
\left\|u_{1}\right\|^{2}+\left\|u_{2}\right\|^{2}+\cdots+\left\|u_{n}\right\|^{2}+\cdots<+\infty .
$$

Assume that a mapping $\Upsilon: \Delta \rightarrow \Delta$ is defined by

$$
\mathrm{G}(\mathrm{u})=(5-\|\mathrm{u}\|) \mathrm{u}, \forall \mathrm{u} \in \Sigma,
$$

where $\Delta=\{u \in \Sigma:\|u\| \leqslant 3\}$. We can easily see that $\Upsilon$ is weakly sequentially continuous on $\Sigma$ and the solution set is $\operatorname{VI}(\Delta, \curlyvee)=\{0\}$. For any $u, y \in \Sigma$, we have

$$
\begin{aligned}
\|\Upsilon(u)-\Upsilon(y)\| & =\|(5-\|u\|) u-(5-\|y\|) y\| \\
& =\|5(u-y)-\| u\|(u-y)-(\|u\|-\|y\|) y\| \\
& \leqslant 5\|u-y\|+\|u\|\|u-y\|+\|u\|-\|y\|\|y\| \\
& \leqslant 5\|u-y\|+3\|u-y\|+3\|u-y\| \\
& \leqslant 11\|u-y\| .
\end{aligned}
$$

Hence $\Upsilon$ is L-Lipschitz continuous with $L=11$. For any $u, y \in \Sigma$, let $\langle\curlyvee(u), y-u\rangle>0$, such that

$$
(5-\|u\|)\langle u, y-u\rangle>0 .
$$

Since $\|u\| \leqslant 3$, it implies that

$$
\langle u, y-u\rangle>0 .
$$

Consider that

$$
\begin{aligned}
\langle\curlyvee(y), y-u\rangle & =(5-\|y\|)\langle y, y-u\rangle \\
& \geqslant(5-\|y\|)\langle y, y-u\rangle-(5-\|y\|)\langle u, y-u\rangle \geqslant 2\|u-y\|^{2} \geqslant 0 .
\end{aligned}
$$

Hence a mapping $\Upsilon$ is quasimonotone on $\Delta$. Let $u=\left(\frac{5}{2}, 0,0, \ldots, 0, \ldots\right)$ and $y=(3,0,0, \ldots, 0, \ldots)$ such that

$$
\langle\curlyvee(u)-\Upsilon(y), u-y\rangle=\left(\frac{5}{2}-3\right)^{3}<0
$$

Consider the following projection formula:

$$
P_{\Delta}(u)= \begin{cases}u, & \text { if }\|u\| \leqslant 3 \\ \frac{3 u}{\|u\|}, & \text { otherwise. }\end{cases}
$$

Figures 1-6 and Table 1 show numerical results. The control conditions are taken in the following way: (i) Algorithm 1 (shortly, Alg01): $\varkappa=\frac{0.7}{\mathrm{~L}}, \vartheta_{\mathrm{n}}=\frac{1}{(\mathrm{n}+2)}, \mathrm{D}_{\mathrm{n}}=\left\|\mathrm{u}_{\mathrm{n}}-\mathrm{u}_{\mathrm{y}}\right\|$; (ii) Algorithm 2 (shortly, Alg02): $\varkappa_{1}=0.22, \chi=0.44, \vartheta_{n}=\frac{1}{(n+2)}, D_{n}=\left\|u_{n}-y_{n}\right\| ;$ (iii) Algorithm 3 (shortly, Alg03): $\varkappa_{1}=0.22, \chi=$ $0.44, \varphi_{n}=\frac{100}{(n+1)^{2}}, \vartheta_{n}=\frac{1}{(n+2)}, D_{n}=\left\|u_{n}-y_{n}\right\|$.

Table 1: Numerical values for Example 4.1.

\begin{tabular}{lccllll}
\hline & \multicolumn{3}{c}{ Number of iterations } & \multicolumn{2}{c}{ Execution time in seconds } \\
\hline & & & & & \\
$\mathfrak{u}_{1}$ & Alg01 & Alg02 & Alg03 & Alg01 & Alg02 & Alg03 \\
\hline$\left(1,1, \cdots, 1_{10000}, 0,0, \cdots\right)$ & 111 & 105 & 91 & 4.3553636 & 6.7229686 & 7.3973612 \\
$(1,2, \cdots, 10000,0, \cdots)$ & 124 & 100 & 87 & 6.2130909 & 9.4322004 & 6.7588432 \\
$\left(8,8, \cdots, 8_{10000}, 0, \cdots\right)$ & 133 & 116 & 101 & 5.4634891 & 7.8256418 & 8.8856192 \\
\hline
\end{tabular}




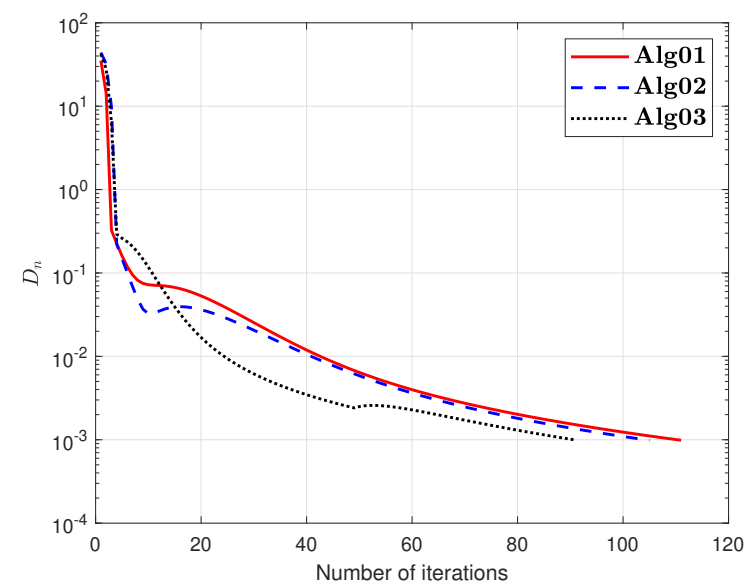

Figure 1: Numerical illustration of Algorithms 1, 2, and 3 while $u_{1}=\left(1,1, \ldots, 1_{10000}, 0,0, \ldots\right)$.

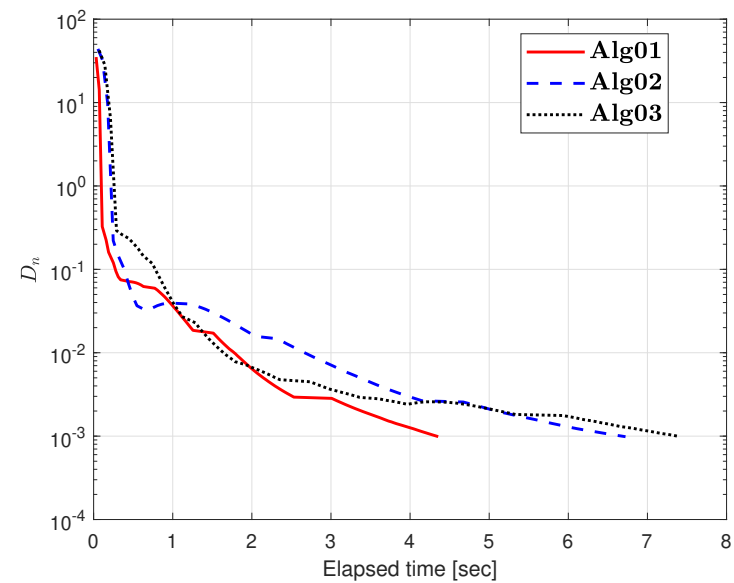

Figure 2: Numerical illustration of Algorithms 1, 2, and 3 while $u_{1}=\left(1,1, \ldots, 1_{10000}, 0,0, \ldots\right)$.

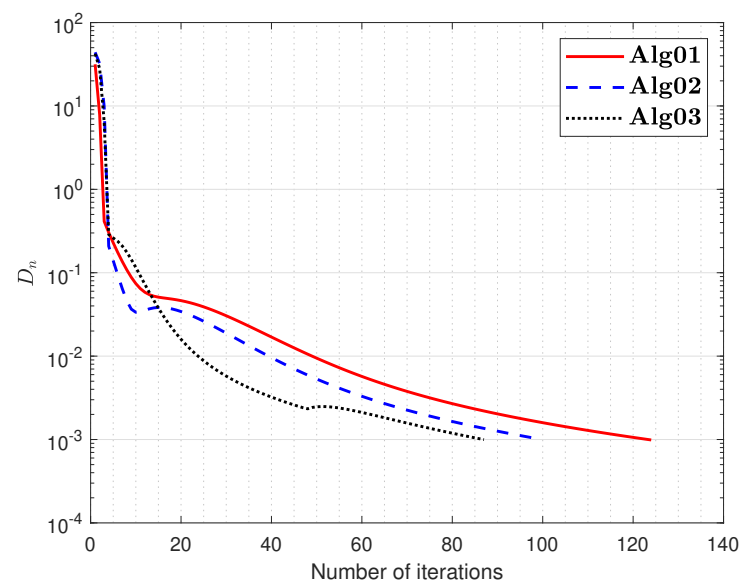

Figure 3: Numerical illustration of Algorithms 1, 2, and 3 while $u_{1}=(1,2, \ldots, 10000,0,0, \ldots)$. 


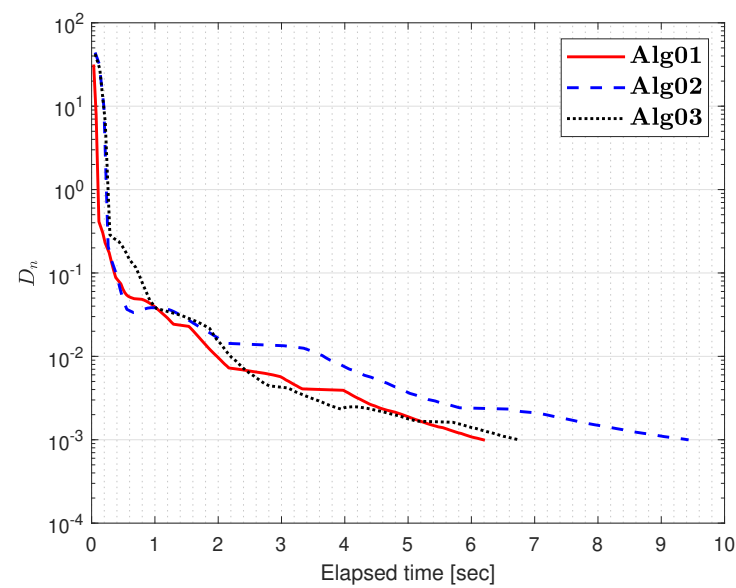

Figure 4: Numerical illustration of Algorithms 1, 2, and 3 while $u_{1}=(1,2, \ldots, 10000,0,0, \ldots)$.

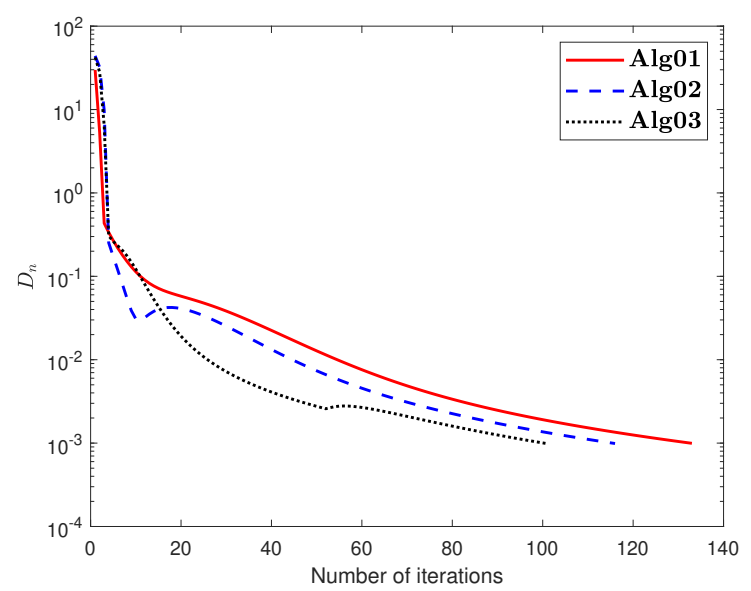

Figure 5: Numerical illustration of Algorithms 1, 2, and 3 while $u_{1}=\left(8,8, \ldots, 8_{10000}, 0,0, \ldots\right)$.

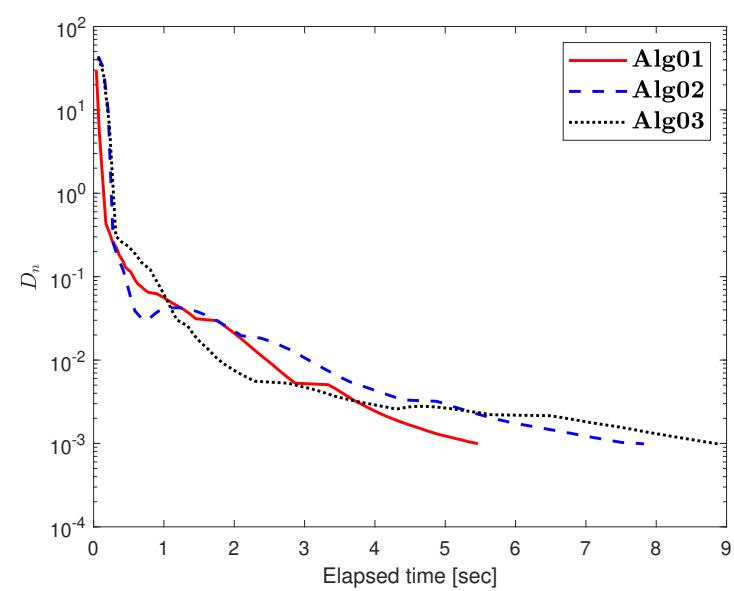

Figure 6: Numerical illustration of Algorithms 1, 2, and 3 while $u_{1}=\left(8,8, \ldots, 8_{10000}, 0,0, \ldots\right)$.

\section{Conclusion}

To find a numerical solution to the quasimonotone variational inequality problems in real Hilbert space, we have developed different modified extragradient-type methods. While following a different 
step size rule, all sequences generated by the proposed method are strongly convergent to the solution. Numerical findings are summarized to demonstrate the numerical effectiveness of our algorithm in comparison to other proposed methods. These numerical studies have indicated that the variable step size rule outperforms the fixed step size rule in most situations.

\section{Acknowledgment}

The authors are grateful to the referees for numerous comments that improved the quality of the paper. The first author was partially supported by Valaya Alongkorn Rajabhat University under the Royal Patronage. The second and third authors would like to thank Phetchabun Rajabhat University.

\section{References}

[1] A. S. Antipin, On a method for convex programs using a symmetrical modification of the lagrange function, Ekonomika i Matem. Metody, 12 (1976), 1164-1173. 1

[2] H. H. Bauschke, P. L. Combettes, Convex Analysis and Monotone Operator Theory in Hilbert Spaces, Springer, New York, (2017). 2.1

[3] L. C. Ceng, Asymptotic inertial subgradient extragradient approach for pseudomonotone variational inequalities with fixed point constraints of asymptotically nonexpansive mappings, Commun. Optim. Theory, 2020 (2020), 21 pages. 1

[4] L. C. Ceng, A. Petruşel, X. Qin, J. C. Yao, A modified inertial subgradient extragradient method for solving pseudomonotone variational inequalities and common fixed point problems, Fixed Point Theory, 21 (2020), 93-108. 1

[5] L. C. Ceng, A. Petrusel, C. F. Wen, J. C. Yao, Inertial-like subgradient extragradient methods for variational inequalities and fixed points of asymptotically nonexpansive and strictly pseudocontractive mappings, Mathematics, 7 (2019), 19 pages. 1

[6] L. C. Ceng, A. Petruşel, J. C. Yao, On Mann viscosity subgradient extragradient algorithms for fixed point problems of finitely many strict pseudocontractions and variational inequalities, Mathematics, 7 (2019), 14 pages. 1

[7] L. C. Ceng, X. Qin, Y. Shehu, J. C. Yao, Mildly inertial subgradient extragradient method for variational inequalities involving an asymptotically nonexpansive and finitely many nonexpansive mappings, Mathematics, 7 (2019), 19 pages. 1

[8] L.-C. Ceng, M.-J. Shang, Hybrid inertial subgradient extragradient methods for variational inequalities and fixed point problems involving asymptotically nonexpansive mappings, Optimization, 70 (2019), 715-740. 1

[9] L. C. Ceng, M. Shang, Composite extragradient implicit rule for solving a hierarch variational inequality with constraints of variational inclusion and fixed point problems, J. Inequal. Appl., 2020 (2020), 19 pages. 1

[10] Y. Censor, A. Gibali, S. Reich, The subgradient extragradient method for solving variational inequalities in Hilbert space, J. Optim. Theory Appl., 148 (2010), 318-335. 1

[11] Y. Censor, A. Gibali, S. Reich, Extensions of Korpelevich's extragradient method for the variational inequality problem in Euclidean space, Optimization, 61 (2012), 1119-1132. 1

[12] J. F. Chu, F. Gharehgazlouei, S. Heidarkhani, A. Solimaninia, Three nontrivial solutions for Kirchhoff-type variationalhemivariational inequalities, Results Math., 68 (2015), 71-91. 1

[13] C. M. Elliott, Variational and quasivariational inequalities applications to free-boundary problems (Claudio baiocchi and António Capelo), SIAM Rev., 29 (1987), 314-315. 1

[14] A. N. Iusem, B. F. Svaiter, A variant of Korpelevich's method for variational inequalities with a new search strategy, Optimization, 42 (1997), 309-321. 1

[15] G. Kassay, J. Kolumbán, Z. Páles, On nash stationary points, Publ. Math. Debrecen 54 (1999), 267-279. 1

[16] G. Kassay, J. Kolumbán, Z. Páles, Factorization of minty and stampacchia variational inequality systems, European J. Oper. Res., 143 (2002), 377-389.

[17] D. Kinderlehrer, G. Stampacchia, An introduction to variational inequalities and their applications, Classics in Applied Mathematics, 31, SIAM, Philadelphia, (2000).

[18] I. Konnov, Equilibrium models and variational inequalities, Elsevier B. V., Amsterdam, (2007). 1

[19] G. M. Korpelevich, The extragradient method for finding saddle points and other problems, Matecon, 12 (1976), 747-756. 1

[20] L. Liu, S. Y. Cho, J. C. Yao, Convergence analysis of an inertial Tseng's extragradient algorithm for solving pseudomonotone variational inequalities and applications, J. Nonlinear Var. Anal., 5 (2021), 627-644. 1

[21] P.-E. Maingé, Strong convergence of projected subgradient methods for nonsmooth and nonstrictly convex minimization, Set-Valued Anal., 16 (2008), 899-912. 2.3

[22] A. Moudafi, Viscosity approximation methods for fixed point problems, J. Math. Anal. Appl., 241 (2000), 46-55. 1

[23] K. Muangchoo, H. ur Rehman, P. Kumam, Two strongly convergent methods governed by pseudo-monotone bi-function in a real Hilbert space with applications, J. Appl. Math. Comput., 67 (2021), 891-917. 1

[24] M. A. Noor, Some iterative methods for nonconvex variational inequalities, Comput. Math. Model., 21 (2010), 97-108. 1 
[25] F. U. Ogbuisi, O. S. Iyiola, J. Ngnotchouye, T. Shumba, On inertial type self-adaptive iterative algorithms for solving pseudomonotone equilibrium problems and fixed point problems, J. Nonlinear Funct. Anal., 2021 (2021), 18 pages. 1

[26] G. Stampacchia, Formes bilinéaires coercitives sur les ensembles convexes, C. R. Acad. Sci. Paris, 258 (1964), $4413-4416$. 1

[27] D. Q. Tran, M. L. Dung, V. H. Nguyen, Extragradient algorithms extended to equilibrium problems, Optimization, 57 (2008), 749-776. 3

[28] P. Tseng, A modified forward-backward splitting method for maximal monotone mappings, SIAM J. Control Optim., 38 (2000), 431-446. 1, 1

[29] H. ur Rehman, A. Gibali, P. Kumam, K. Sitthithakerngkiet, Two new extragradient methods for solving equilibrium problems, Rev. R. Acad. Cienc. Exactas Fís. Nat. Ser. A Mat. RACSAM, 115 (2021), 25 pages. 1

[30] H. ur Rehman, P. Kumam, A. B. Abubakar, Y. J. Cho, The extragradient algorithm with inertial effects extended to equilibrium problems, Comput. Appl. Math., 39 (2020), 26 pages.

[31] H. ur Rehman, P. Kumam, Y. J. Cho, P. Yordsorn, Weak convergence of explicit extragradient algorithms for solving equilibirum problems, J. Inequal. Appl., 1 (2019), 25 pages.

[32] H. ur Rehman, P. Kumam, Q.-L. Dong, Y. J. Cho, A modified self-adaptive extragradient method for pseudomonotone equilibrium problem in a real Hilbert space with applications, Math. Methods Appl. Sci., 44 (2020), 3527-3547.

[33] H. ur Rehman, P. Kumam, A. Gibali, W. Kumam, Convergence analysis of a general inertial projection-type method for solving pseudomonotone equilibrium problems with applications, J. Inequal. Appl., 2021 (2021), 27 pages. 1

[34] H.-K. Xu, Another control condition in an iterative method for nonexpansive mappings, Bull. Austral. Math. Soc., 65 (2002), 109-113. 2.2

[35] J. Yang, H. Liu, A modified projected gradient method for monotone variational inequalities, J. Optim. Theory Appl., 179 (2018), 197-211. 1

[36] J. Yang, H. W. Liu, Z. X. Liu, Modified subgradient extragradient algorithms for solving monotone variational inequalities, Optimization, 67 (2018), 2247-2258. 1

[37] P. Yordsorn, P. Kumam, H. ur Rehman, Modified two-step extragradient method for solving the pseudomonotone equilibrium programming in a real Hilbert space, Carpathian J. Math., 36 (2020), 313-330. 1

[38] P. Yordsorn, P. Kumam, H. ur Rehman, A. H. Ibrahim, A weak convergence self-adaptive method for solving pseudomonotone equilibrium problems in a real Hilbert space, Mathematics, 8 (2020), 24 pages. 1

[39] L. X. Zhang, C. J. Fang, S. L. Chen, An inertial subgradient-type method for solving single-valued variational inequalities and fixed point problems, Numer. Algorithms, 79 (2018), 941-956. 1

[40] T.-Y. Zhao, D.-Q. Wang, L.-C. Ceng, L. He, C.-Y. Wang, H.-L. Fan, Quasi-inertial Tseng's extragradient algorithms for pseudomonotone variational inequalities and fixed point problems of quasi-nonexpansive operators, Numer. Funct. Anal. Optim., 42 (2021), 69-90. 1 